In this way, a dynamically consistent global climate reconstruction can be retrieved from patchy data.

Progress in understanding the mechanisms that lead to both millennialscale natural climate variability and its spatial patterns depends at least partly on the ability of climate models to simulate all the relevant processes. As part of the ongoing assessment by the Intergovernmental Panel on Climate Change, the next generation of climate models will be challenged with the task of realistically simulating the processes that have determined the climate of the past millennium.

Johann H. Jungclaus is at the Max Planck Institute for Meteorology, Bundesstrasse 53, 20146 Hamburg, Germany.

e-mail: johann.jungclaus@zmaw.de

References

1. Mann, M. E., Bradley, R. S. \& Hughes, M. K. Nature 392, 779-787 (1998).

2. Jansen, E. J. et al. in IPCC Climate Change 2007: The Physical Science Basis (eds Solomon, S. et al.) 433-497 (Cambridge Univ. Press, 2007).
3. National Research Council Surface Temperature Reconstructions for the Last 2,000 Years (Natl Acad. Press, 2006).

4. http://meetingorganizer.copernicus.org/EGU2009/oral_ programme $/ 218$

5. Trouet, V. et al. Science 324, 78-80 (2009).

6. Cobb, K. M., Charles, C. D., Cheng, H. \& Edwards, R. L. Nature 424, 272-276 (2003)

7. Emile-Geay, J., Cane, M. A., Seager, R., Kaplan, A. \& Almasi, P. Paleoceanography 22, PA3210 (2007)

8. Mann, M. E., Cane, M. A., Zebiak, S. E. \& Clement, A. J. Clim 18, 447-456 (2005).

9. Meehl, G. A. et al. in IPCC Climate Change 2007: The Physical Science Basis (eds Solomon, S. et al.) 747-845 (Cambridge Univ. Press, 2007).

10. Lean, J. PAGES Newsletter 13, 13-15 (2005).

\title{
ERRATUM
}

\section{Insight despite imperfection}

\section{Mat Collins}

Nature Geoscience 2, 315-316 (2009); published online: 30 April 2009; corrected after print 2 June 2009.

In this News \& Views, Figure 1 incorrectly shows an Antarctic iceberg rather than Arctic sea-ice as stated in the caption. 